\title{
Effect of combined laser dyes on the efficiency of cholesteric liquid crystal lasers
}

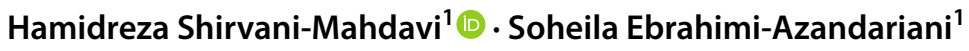

Received: 26 August 2018 / Accepted: 15 January 2019 / Published online: 22 January 2019

(c) The Author(s) 2019

\begin{abstract}
A high efficiency band-edge cholesteric liquid crystal (CLC) laser comprising an optimal binary-dye mixture (OBD) with $62 \mathrm{wt} \%$ DCM and $38 \mathrm{wt} \%$ PM597 as the active medium is scrutinized. The measurements indicate that both the fluorescence spectrum width and the order parameter of OBD in the host of nematic liquid crystal (BL009) enhance compared with those for each individual dye. Furthermore, at the fluorescence peak, the optical efficiency of the PM597-doped CLC laser is $\sim 1.5 \times$ higher than that of DCM, and the laser emission energy for OBD-doped CLC between the wavelengths $595 \mathrm{~nm}$ and $613 \mathrm{~nm}$ is even higher than that of PM597-doped CLC, so that at the maximum fluorescence of OBD, $\lambda \approx 605 \mathrm{~nm}$, the improvement is over $20 \%$.
\end{abstract}

Keywords Binary-dye mixture $\cdot$ Band-edge laser $\cdot$ Fluorescence spectrum $\cdot$ Slope efficiency

\section{Introduction}

Cholesteric liquid crystals (CLCs) exhibit a natural and selforganized periodic helical structure. This periodicity gives rise to a periodic modulation of the refractive index and consequently a one-dimensional photonic band gap (PBG) [1-6], which in turn inhibits the propagation of certain frequency range of electromagnetic radiation [7]. The frequency range of the PBG is determined by the pitch $(P)$ and the ordinary $\left(n_{\mathrm{o}}\right)$ and extraordinary $\left(n_{\mathrm{e}}\right)$ refractive indices of CLC. The reflection band edges occur at $\lambda_{\mathrm{s}}=n_{\mathrm{o}} P$ and $\lambda_{1}=n_{\mathrm{e}} P$ where $s$ and $l$ specify short- and long-wavelength edges, respectively [8]. Hence, a CLC doped with a laser dye can be operated as a band-edge laser [9-11]. Lasing in such materials was firstly proposed about 38 years ago [12] and has attracted a widespread attention for its significant efficiency. Since the advent of CLC lasers, extensive research has been carried out to enhance the laser efficiency and reduce the energy threshold [13-16].

Different factors, including birefringence, order parameter and repetition rate of stimulating laser, are effective on the

Hamidreza Shirvani-Mahdavi

hsm@iauctb.ac.ir

1 Photonics Research Laboratory, Department of Physics, Islamic Azad University (Central Tehran Branch), Tehran, Iran efficiency of CLC lasers. A high orientational order parameter for the liquid crystal as the host of CLC laser leads to a notable birefringence $[17,18]$, which in turn decreases the threshold energy and increases the slope efficiency [19, 20]. On the other hand, laser dyes with a large order parameter of the transition dipole moment cause more absorption of the pumping energy which consequently results in an optimal performance of CLC lasers.

Araoka et al. [21] showed that conjugated polymeric dyes having a triptycene structure can highly be solved in liquid crystals, which results in suppressing the fluctuation of liquid crystal molecules, a higher order parameter and eventually a better efficiency. Shin et al. [22] also indicated that polymer dyes are highly aligned along the local director of liquid crystal, leading to a higher efficiency. Dolgaleva et al. [23] proposed a highly efficient organic dye, oligofluorene, for lasing in cholesteric monomeric liquid crystal oligomers. Oligofluorene-doped CLC laser produced maximum output energy because of its superior temporal and spatial stability. By using oligo-thiophene (OT) derivative having order parameter of about 0.65 , lasing occurs in a DMQTT-doped CLC laser at only the low-energy edge of the PBG above the threshold of $4.6 \mu \mathrm{J} \mathrm{cm}-2 /$ pulse [24]. This lasing emission mode selection is attributed to the high order parameter of OT molecules.

In the present study, in order to enhance the optical efficiency and decrease the threshold excitation energy, a 
binary-dye-dope liquid crystal, including 68 wt\% DCM and $32 \mathrm{wt} \%$ PM597, is utilized. The measurement indicates that the order parameter of this optimal binary-dye mixture (OBD) is markedly increased by 0.55 in comparison with that of constituent dyes, DCM (0.33) and PM597 (0.45). In addition, the width of binary-dye fluorescence spectrum undergoes an increase of about 25 percent compared to that of its components. The results obtained from an OBDdoped CLC laser fabricated in the laboratory emphasize the enhancement of slope efficiency and the reduction of energy threshold. Furthermore, to prove the potentially tunability of OBD-doped CLC lasers, five CLC lasers with similar concentration of OBD and different concentrations of chiral agent, corresponding to various laser wavelengths, are prepared and experimented.

\section{Experimental investigation}

\section{Laboratory setup}

Figure 1 depicts the setup used for the CLC laser in this research. The pump source is a frequency-doubled, Q-switched, Nd:YAG pulsed laser $(\lambda=532 \mathrm{~nm}$, from Continuum) with pulse duration of $6 \mathrm{~ns}$. All the measurements were taken at $1 \mathrm{~Hz}$ laser repetition rate in order to reduce the accumulated thermal effect originating from dye absorption.

An attenuator was used to set the pumping energy to our desired value. A beam splitter divides the pumping laser light into two equal parts so that one, as the measuring ray, propagates toward the first energy meter EM1 (Gentec) and the other, as the pumping ray, excites the active CLC. The linear polarizer and the quarter-wave plate were used to create a left-handed circular polarization (LCP) to avoid the reflection from the stop band of the CLC film. The slope efficiency and the threshold energy are both dependent on the spot size of pump beam [25] which is adjusted by lens $\mathrm{L} 1$. The spot size was chosen to be $65 \mu \mathrm{m}$. The lens L2 was utilized to focus the output laser emission from the CLC

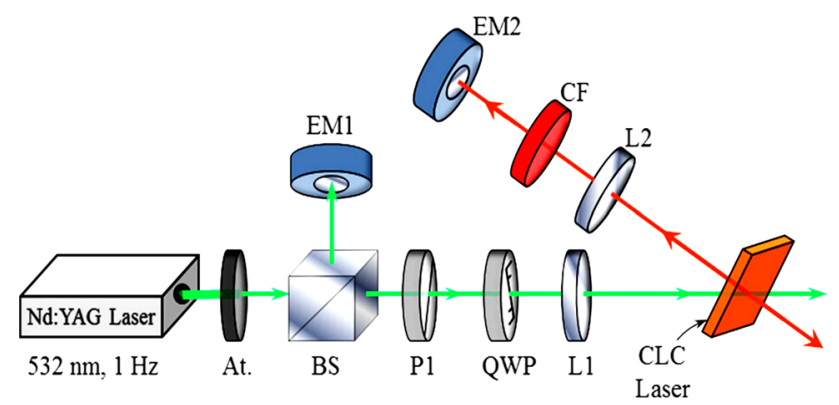

Fig. 1 Experimental setup: At attenuator, $B S$ beam splitter, $P 1$ polarizer, $Q W P$ quarter-wave plate, $L 1$ and $L 2$ lenses, $C F$ color filter, $E M 1$ and $E M 2$ energy meters sample on the second energy meter EM2 (Gentec). Furthermore, in order to eliminate useless lights with wavelengths except CLC laser wavelength, the colored filter was used.

\section{Sample preparation}

We classified our cells into five groups. The first one contains two cells with the similar high birefringence nematic liquid crystal BL009 $\left(n_{\mathrm{e}}=1.8098, n_{\mathrm{o}}=1.5288\right.$ and $\Delta n=0.281$ from Merck) and the left-handed chiral dopant MLC6247 (helical twisting power $=11.3(\mu \mathrm{m} w \mathrm{t} \%)^{-1}$ from Merck) but different laser dyes DCM [(4-(dicyanomethylene)-2methyl-6-(4-dimethlyaminostryl)-4H-pyran)] and PM597 [1,3,5,7,8-pentamethyl-2,6-di-t-butylpyrromethene-difluoroborate complex] both from Exciton. The amount of chiral agent is adjusted in a way that the long-wavelength edge of the PBG matches with the fluorescence peak of each dye. This group was prepared to measure the laser wavelength of each dye individually. The second group involving six cells with different concentrations of binary-dye mixture (DCM + PM597) in the host BL009 was prepared in order to achieve the optimal ratio of two dyes. The third group includes one cell in which $1.5 \mathrm{wt} \%$ of OBD was added to CLC (BL009+ MLC6247) in order to measure its transmission and laser line spectra. The fourth group comprising nine OBD-doped CLCs with different concentrations of chiral agent was fabricated to find the best laser emission energy versus wavelength in this type of laser. To measure the order parameter of the dyes DCM, PM597 and OBD, three cells (as the fifth group) were fabricated with the same host (BL009). All mixtures were thoroughly mixed before they were capillary-filled into the homogeneous LC cells with $10 \mu \mathrm{m}$ thickness in the isotropic state (at $105^{\circ} \mathrm{C}$ ). After a slow cooling process $\left(0.3^{\circ} \mathrm{C} \mathrm{min}^{-1}\right)$, a defect-free singledomain cholesteric planar structure was formed.

\section{Results and discussion}

In DCM dye, the quantum yields of the formation of triplet states and the cis-trans photo-isomerization of the double bond are, respectively, less than 0.003 and 0.007 [26]. This means that in DCM compared with some other dyes, less percentage of pumping energy converts to heat, leading to less energy loss and consequently an enhancement of the slope efficiency. Hence, in most CLC lasers, DCM is mainly used as the active material.

But the slope efficiency of CLC lasers, in addition, depends on the amount of pumping energy absorption by the dye used. For instance, measurements indicate that the absorption peak of DCM in the presence of the cholesteric host [BL009+ MLC6247 (25.40 wt\%)] is $480 \mathrm{~nm}$ (Fig. 2) while for PM597 in an approximately similar matrix (with 


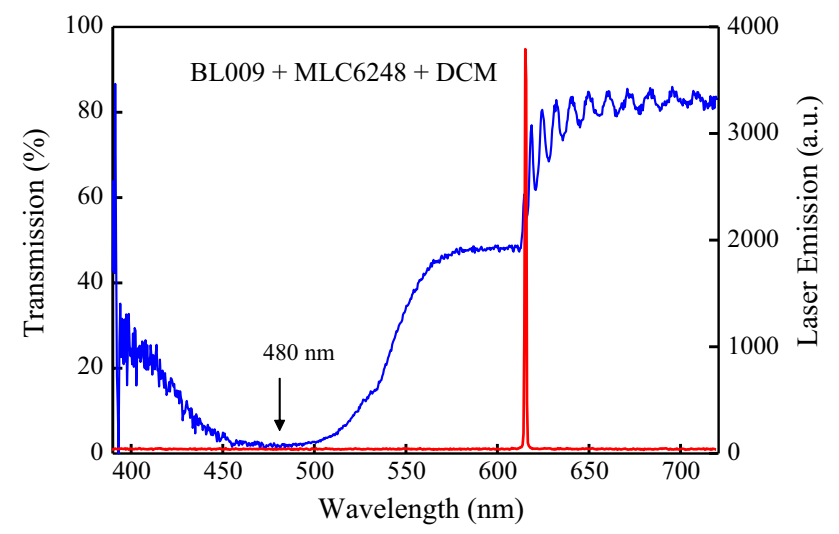

Fig. 2 Blue line: transmission spectrum of DCM-doped cholesteric liquid crystal, red line: laser emission at the long band-edge wavelength with $15 \mu \mathrm{J} /$ pulse and $532 \mathrm{~nm}$ pumping energy and wavelength, respectively. Both spectra were measured by an HR2000 spectrometer (Ocean Optics)

$24.77 \mathrm{wt} \%$ of the chiral impurity MLC6247) the maximum absorption according to Fig. 3 occurs at $531 \mathrm{~nm}$.

Therefore, if these two active CLCs have been pumped through a frequency-doubled, Q-switched, Nd:YAG pulsed laser with $\lambda=532 \mathrm{~nm}$, since maximum absorption of $\operatorname{PM} 597\left(\lambda_{\mathrm{p}-\mathrm{abs}}=531 \mathrm{~nm}\right)$ is much closer to this wavelength, despite the very low loss energy of DCM, a PM597 dyedoped CLC laser can emit more energy. We will discuss this issue after describing Fig. 3 which depicts the transmission spectrum of a PM597-doped CLC. In order to avoid overlap of the photonic band gap and the absorption region of PM597, the concentration of chiral impurity in the sample was selected to be an appropriate amount so that both the long- and short-wavelength edges of the stop band can be clearly observed. On the other hand, the fluorescence

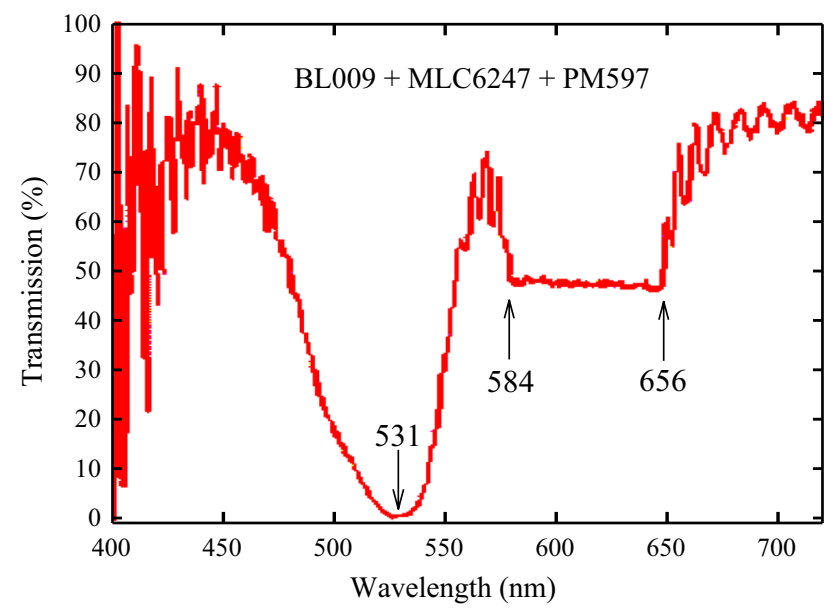

Fig. 3 Transmission spectrum of PM597-doped cholesteric liquid crystal [BL009+MLC6247 (24.77 wt\%)]. According to Fig. 4, no lasing can occur at the short and long band-edge wavelengths spectrum of PM597 in the host of nematic liquid crystal BL009 (Fig. 4) measured by a PTI QuantaMaster spectrofluorometer indicates that the probability of lasing in the laser dye PM597 is remarkable only for wavelengths between $\lambda_{1-f l u}=563 \mathrm{~nm}$ and $\lambda_{\mathrm{r}-\mathrm{flu}}=621 \mathrm{~nm}$. Accordingly, in this case, since the short-wavelength edge of PBG, $\lambda_{\mathrm{S}}=584 \mathrm{~nm}$ (Fig. 3), is so close to the fluorescence peak $\left(\lambda_{\mathrm{p}-\mathrm{flu}}=590 \mathrm{~nm}\right)$, it is expected that the laser emission at $\lambda_{\mathrm{S}}$ is much more probable than $\lambda_{\mathrm{L}}=656 \mathrm{~nm}$. But PM597, similar to DCM, has positive birefringence, and thus, the alignment of the laser dye molecules is perpendicular to the light polarization in this edge which implies that the probability of laser emission at this wavelength is extremely low.

To solve the problem, the chiral impurity must so be increased that the long-wavelength edge $\left(\lambda_{L}\right)$ of stop band shifts toward the peak fluorescence of laser dye PM597. Wherever $\lambda_{\mathrm{L}}$ grows to be $\lambda_{\mathrm{p}-\text { flu }}$, the maximum laser energy will occur. In order to compare CLC lasers doped with DCM and PM597 dyes, two active CLC samples were prepared with the specifications tabulated in Table 1. The amount of chiral impurity in each sample was selected so that the long-wavelength edge coincides with the fluorescence peak of its corresponding laser dye $\left(\lambda_{\mathrm{DCM}-\mathrm{fl.P}}=608 \mathrm{~nm}\right.$, $\lambda_{\text {PM597-fl.P }}=590 \mathrm{~nm}$ ).

Measurements show that the laser emission energy in PM597-doped CLC laser is about $1.5 \times$ higher than that in DCM-doped CLC laser. This event may occur as a result of different reasons. First of all as formerly discussed, the pumping wavelength, $532 \mathrm{~nm}$, concurs with the absorption peak wavelength of PM597, which in turn leads to noticeable amount of the pumping energy absorption in comparison with DCM. Furthermore, the order parameter of the laser dye transition dipole moment can be an effective factor in

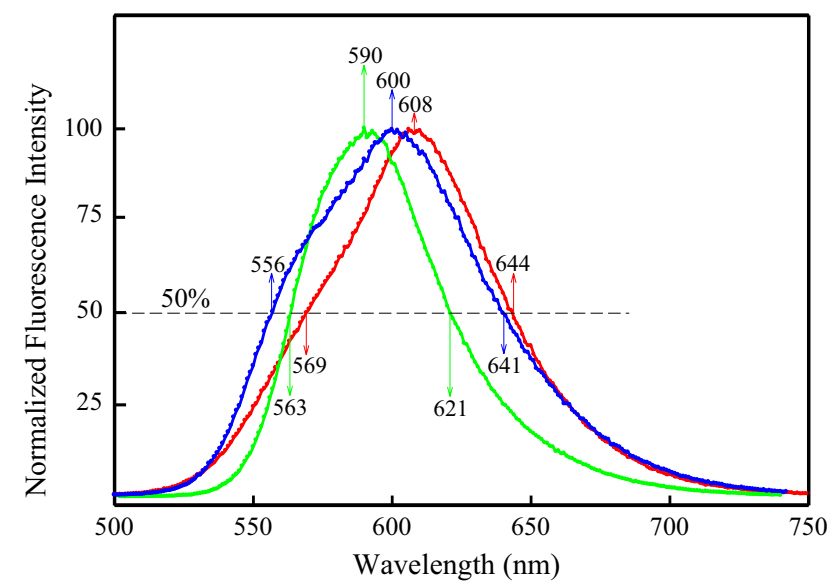

Fig. 4 Fluorescence spectra of three laser dyes: PM597 (green line), DCM (red line) and OBD (blue line), in the host of nematic liquid crystal BL009 measured by a QuantaMaster spectrofluorometer (from Photon Technology International) 
Table 1 Information of two CLC doped with DCM and PM597 in order to compare their performance

\begin{tabular}{lllll}
\hline Type of lasers & NLC host & Chiral impurity (wt\%) & Laser dye (wt\%) & $\begin{array}{l}\text { Laser } \\
\text { wavelength } \\
\text { (nm) }\end{array}$ \\
\hline DCM-doped CLC laser & BL009 & MLC6247 24.54 & DCM 1.5 & 608 \\
PM597-doped CLC laser & BL009 & MLC6247 27.83 & PM597 1.5 & 590 \\
\hline
\end{tabular}<smiles>[14CH3]N([O])c1ccc(C=CC2=CC(=C(C#N)C#N)C=C([Ge])O2)cc1</smiles>

Fig. 5 Chemical structure of the laser dye DCM

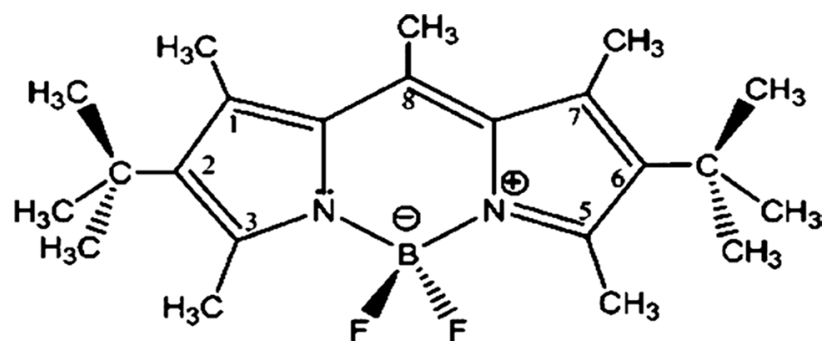

Fig. 6 Chemical structure of the laser dye pyrromethene 597 (PM597)

this phenomenon. Accurate study on fluorescence spectra of PM597 and DCM (Fig. 4) in the nematic liquid crystal host BL009 and their laser performance reminds us that the combination of these two dyes in the same host can probably create much better laser efficiency than two former lasers.

But before addressing this issue, it is important that the optimal concentrations of DCM and PM597 in the CLC mixture [BL009+ MLC6247 ( 25 wt\%)] are determined. For this purpose, first of all, we should consider the chemical structures of DCM and PM597 (Figs. 5, 6). As seen, due to noncovalent interactions between the aromatic rings (quadrupoles) arising from $\pi$ bonds, it is possible that the molecules of dyes DCM and PM-597 can attract each other. This is known as $\pi-\pi$ stacking, which can be caused a molecular aggregation depending on the relative concentration of each dye in the binary-dye mixture and on the total concentration. Similarly, cation $-\pi$ interaction which is also a noncovalent molecular interaction between the face of an electron-rich $\pi$ system (e.g., a benzene quadrupole) and an adjacent cation can produce the same effect. It should be noted that this problem not only occurs in the combination of the two dyes but also may take place in the DCM and PM-597, individually. Theoretically, the study on these phenomena may significantly be complicated so that it can be investigated in an independent research. In practice, in order to overcome this problem, the threshold energy and the slope efficiency were measured for different concentrations of the dye from 0.1 to $3 \mathrm{wt} \%$ for both DCM-doped and PM597-doped CLC lasers. The measurements indicated that for both dyes the concentrations between 1.2 and $1.8 \mathrm{wt} \%$ in a similar CLC mixture lead to the minimum threshold energy and the maximum laser efficiency. To this reason, the concentration of $1.5 \mathrm{wt} \%$ was selected for each of the dyes as an optimal concentration. On the other hand, the planar texture of dye-doped CLC with $1.5 \mathrm{wt} \%$ concentration of each dye observed from a polarized optical microscope (Olympus BX51), without any defect lines, confirms that no aggregation has occurred.

To investigate the effect of combining DCM and PM597 on the laser efficiency, this point must be taken into consideration that since the maximum absorption wavelength of PM597 $(531 \mathrm{~nm})$ is so close to the pumping wavelength, $532 \mathrm{~nm}$, it may behave more effective than DCM at that wavelength. This means that a CLC laser consisting of equal concentrations of these two dyes cannot most probably have an optimal performance. In other words, it appears that DCM, in comparison with PM597, should possess a greater concentration in the composition. With this point of view, different combinations of DCM and PM597 with six weight percents 50-50, 55-45, 60-40, 65-35, 70-30 and 75-25 were prepared, and then, the fluorescence spectrum of each binary-dye mixture was measured in the host of nematic liquid crystal BL009. Then, six CLC lasers with these six binary-dye mixtures were designed and fabricated in a way that the wavelength of each laser matches with its corresponding fluorescence peak. The measurement of emission energy of these lasers proved that two mixtures of 60-40 and 65-35 have better efficiencies. More accurate study on the concentrations of these two dyes specified that the best efficiency of emission energy is associated with the mixture of DCM (62 wt\%) and PM597 (38 wt\%). Furthermore, although the contribution of the two dyes to the binary-dye mixture is not identical, an experiment similar to what was conducted for DCM and PM597 reflects that 
the optimal concentration of OBD in the host BL009 is also about $1.5 \mathrm{wt} \%$.

Figure 4, in addition to the fluorescence spectra of DCM and PM597 in the host of nematic liquid crystal BL009, depicts that of optimal binary-dye mixture (OBD) in the same host. As observed, for OBD, the fluorescence peak is at $600 \mathrm{~nm}$, and the possibility of laser emission is considerable in the range of $\lambda_{1}=556 \mathrm{~nm}$ to $\lambda_{\mathrm{r}}=641 \mathrm{~nm}$. In order to investigate the laser performance of OBD, a dye-doped CLC including the chiral agent MLC6247, the nematic liquid crystal BL009 and $1.5 \mathrm{wt} \%$ of OBD was so prepared that the long-wavelength edge of PBG coincides with the fluorescence peak $\sim 600 \mathrm{~nm}$. Then, a CLC laser was fabricated by injecting the mixture in a planar cell with $10 \mu \mathrm{m}$ thickness. Figure 7 indicates the transmission and laser emission spectra of the OBD-doped CLC laser.

As seen, the absorption peak of OBD (corresponding to zero transmission in the range of $450-540 \mathrm{~nm}$ ) is dramatically wider than that of DCM and PM597, so that the pumping energy (with $\lambda=532 \mathrm{~nm}$ ) is significantly absorbed in this mixture. In addition, CLC laser emits a radiation at $\lambda=601.47 \mathrm{~nm}$ which is close to the fluorescence peak of OBD. The measurement of the laser emission energy in the pumping energy of $25 \mu \mathrm{J} /$ pulse showed that the efficiency at this wavelength is $20 \%$ and $75 \%$ higher than that in PM597and DCM-doped CLC lasers, respectively. A more accurate study on the laser performance of OBD indicated that between the wavelengths $593 \mathrm{~nm}$ and $613 \mathrm{~nm}$, the laser emission energy for OBD-doped CLC laser is higher than that for PM597 one, and this energy difference approaches to its maximum value $(1.2 \times$ PM597 laser energy) at $\sim 602 \mathrm{~nm}$. Higher efficiency of OBD-doped CLC laser may be because of better alignment of DCM and PM597 transition dipole moments with the local director of CLC as a result of effective mutual interaction between two dyes in the liquid crystal

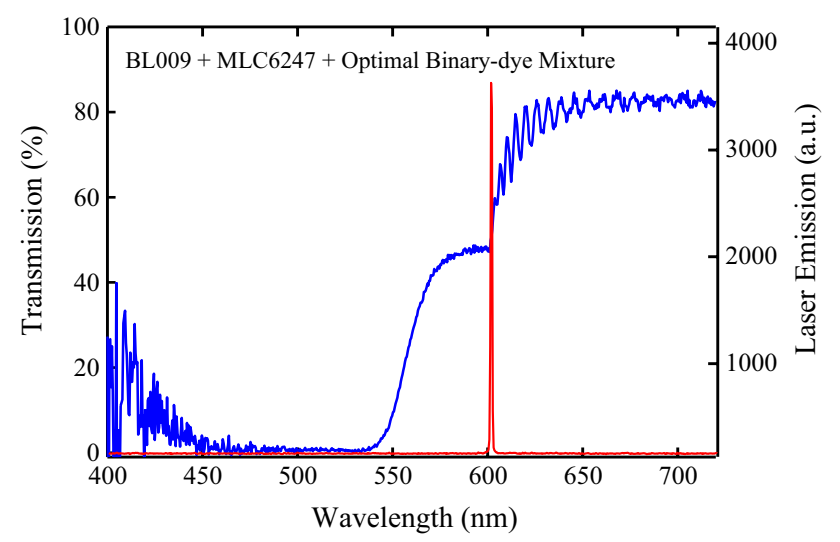

Fig. 7 Blue line: transmission spectrum of OBD-doped cholesteric liquid crystal; red line: laser emission at the long band-edge wavelength with $25 \mu \mathrm{J} /$ pulse pumping energy. Both spectra were measured by an HR2000 spectrometer (Ocean Optics) host. To prove this averment, the order parameter $\left(S_{\mathrm{td}}\right)$ of three laser dyes: DCM, PM597 and OBD, can be calculated by the following equation [27]:

$S_{\mathrm{td}}=\frac{n_{\mathrm{o}} I_{\|}-n_{\mathrm{e}} I_{\perp}}{n_{\mathrm{o}} I_{\|}+2 n_{\mathrm{e}} I_{\perp}}$

where $I_{\|}$and $I_{\perp}$ are the fluorescence intensities emitted from a dye-doped nematic liquid crystal parallel with and perpendicular to the local director of the liquid crystal, respectively. Based on the exact reasoning given in reference 27 , Eq. (1) is always correct to calculate order parameter of the transition dipole moment of a laser dye. For nematic liquid crystals with a very small birefringence $(\Delta n \ll 1), n_{\mathrm{e}} \approx n_{\mathrm{o}}$ and the order parameter can also be evaluated by an equation to some extent different [18], that is,

$S_{\mathrm{td}} \approx \frac{n_{\mathrm{e}} I_{\|}-n_{\mathrm{o}} I_{\perp}}{n_{\mathrm{e}} I_{\|}+2 n_{\mathrm{o}} I_{\perp}}$

Nevertheless, it should be noted that in the case of high birefringence nematic liquid crystals, such as that has been used in this research (BL009 with $\Delta n=0.281$ ), Eq. (2) is no longer perfectly correct and the results obtained from Eq. (1) are definitely more reliable. Figure 8 indicates a suitable setup to measure $I_{\|}$and $I_{\perp}$. The nematic liquid crystal BL009 was used as the host, and the detection of
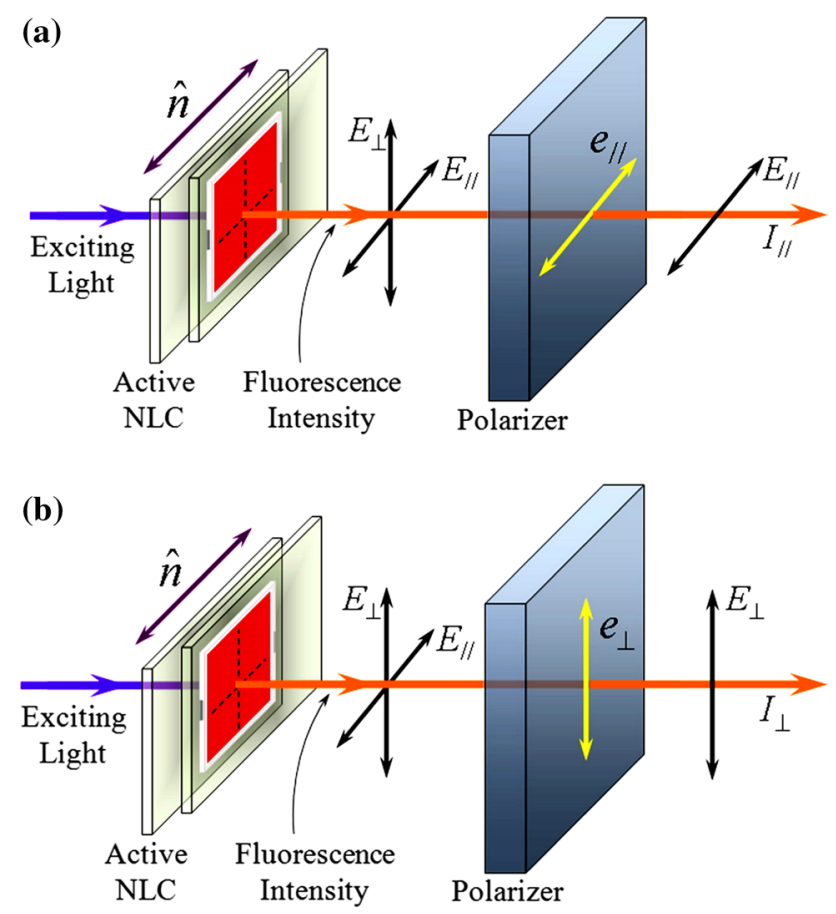

Fig. 8 Measurement approach of intensities related to the two normal propagation modes in a dye-doped nematic liquid crystal. a Parallel with the director $\left(I_{\|}\right)$. b Perpendicular to the director $\left(I_{\perp}\right)$ 
$I_{\|}$and $I_{\perp}$ was carried out by a PTI QuantaMaster spectrofluorometer at the ambient temperature $\left(23{ }^{\circ} \mathrm{C}\right)$. The concentration of each dye in the dye-doped BL009 mixture was adjusted to be $1.5 \mathrm{wt} \%$. In addition, the exciting wavelength for each mixture was selected equal to the wavelength of its maximum absorption.

Figure 9 demonstrates the fluorescence spectra of the three dyes. The average values of $I_{\|}$and $I_{\perp}$ at the wavelength of maximum fluorescence, the exciting wavelengths and the order parameters calculated by Eq. (1) are shown in Table 2. As seen, despite noticeable errors of the measurement especially for DCM due to entered noise, the difference in order parameter of the three dyes is evident. Particularly, the order parameter of OBD has significantly enhanced in comparison with those of DCM and PM597, confirming better alignment of the transition dipole moment of the dyes DCM and PM597 in the OBD-doped liquid crystal compared to that of them singly. Moreover, apart from remarkable absorption of pumping beam by PM597, greater order parameter of PM597 in comparison with DCM is another reason for higher slope efficiency of PM597-doped CLC laser than DCM one.

In addition to the aforementioned points, there is another phenomenon that can be claimed to be the reason for increasing the efficiency of OBD-doped CLC laser. In other words, it may be argued that the fluorescence resonance energy transfer (FRET) between PM597 molecules in the excited state and DCM molecules in the ground state is the reason to enhance the laser efficiency and reduce the threshold energy in OBD-doped CLC laser, as Sonoyama et al. [28] demonstrated about the dyes C153 and DCM in 2008. But in the case of OBD mixture this cannot be true, because the rate of energy transfer dramatically depends upon the extent of spectral overlap of the emission spectrum of the donor with the absorption spectrum of the acceptor; whereas as seen from Figs. 2 and 4, the fluorescence spectrum of PM597 with the width between $550 \mathrm{~nm}$ and $650 \mathrm{~nm}$ does not have any serious overlap with the absorption spectrum of DCM with the width between 400 and $550 \mathrm{~nm}$.

Overall, it is worth mentioning that the combination of PM597 and DCM, for three reasons, is suitable and deserving:

1. The coincidence of the absorption peak wavelength $(531 \mathrm{~nm})$ of PM597 with the pumping wavelength $(532 \mathrm{~nm})$.

2. Good solubility of both PM597 and DCM in the liquid crystal host, BL009.

3. The slight overlap of fluorescence spectrum of PM597BL009 and the absorption spectrum of DCM-BL009, which prevents the fluorescence resonance energy transfer between these two dyes, leads to a simultaneous
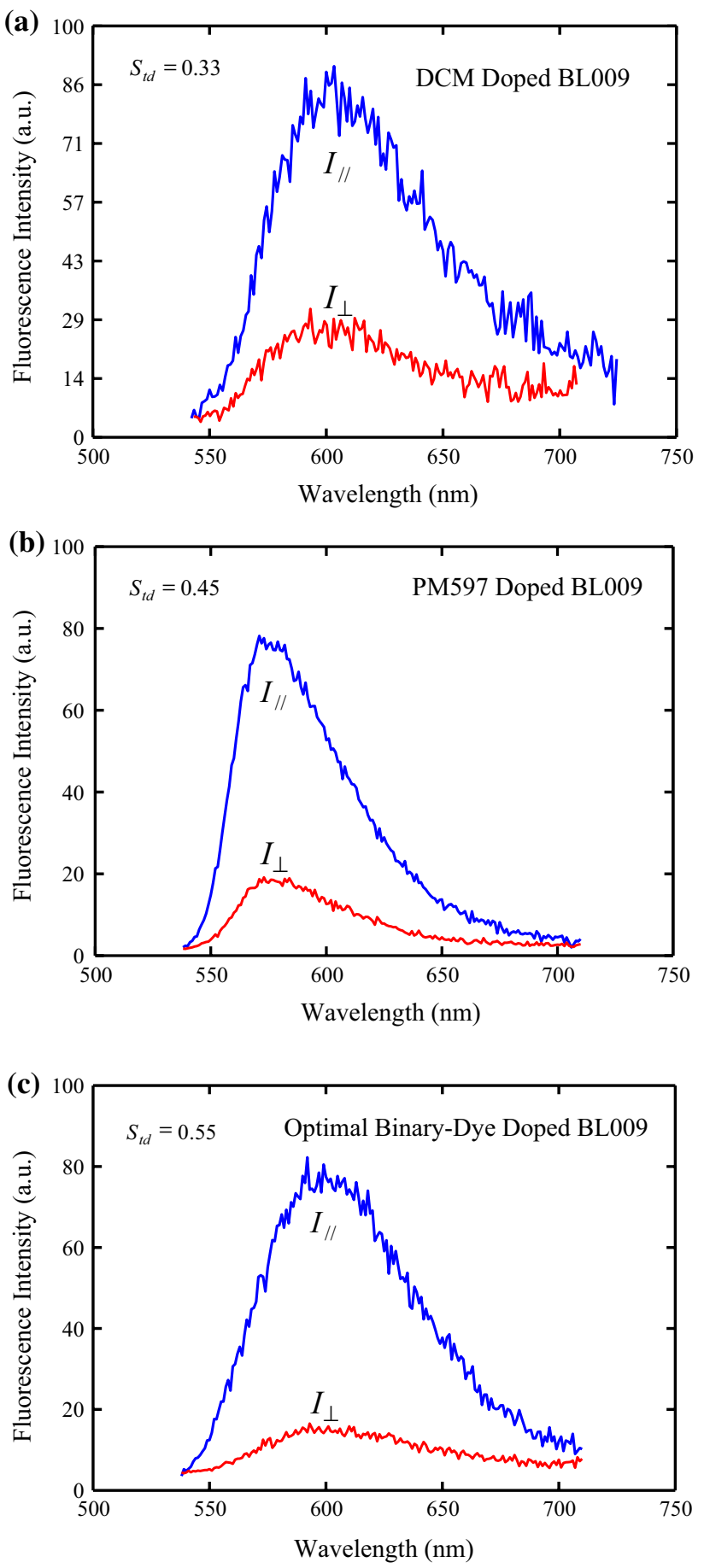

Fig. 9 Normalized fluorescence intensities of a DCM, b PM597 and c OBD in the solvent of BL009 parallel with and perpendicular to the local director of liquid crystal. All measurements were taken by a QuantaMaster spectrofluorometer (from Photon Technology International) 
Table 2 Average values of $I_{\|}$ and $I_{\perp}$ at the wavelength of maximum fluorescence for DCM-, PM597- and OBDdoped BL009 resulted from spectra of Fig. 9

\begin{tabular}{llccr}
\hline Sample & $\begin{array}{l}\text { Exciting wavelength } \\
(\mathrm{nm})\end{array}$ & $\bar{I}_{\|(\mathrm{Max})}$ (counts) & $\bar{I}_{\perp(\mathrm{Max})}$ (counts) & $S_{\mathrm{td}}$ \\
\hline BL009+DCM & 480 & 45,086 & 15,186 & $0.33 \pm 0.06$ \\
BL009+ PM597 & 530 & 39,128 & 9524 & $0.45 \pm 0.02$ \\
BL009+ OBD & 500 & 41,243 & 7573 & $0.55 \pm 0.03$ \\
\hline
\end{tabular}

The order parameters are calculated by Eq (1) and considering the values of $n_{\mathrm{e}}=1.8098$ and $n_{\mathrm{o}}=1.5288$. The measuring temperature was $23{ }^{\circ} \mathrm{C}$, and the concentration of dye in each sample was adjusted to be $1.5 \mathrm{wt} \%$
Table 3 Concentration of constituent materials of five OBD-doped CLC lasers including $1.49 \mathrm{wt} \%$ of OBD

\begin{tabular}{lll}
\hline OBD-doped CLC laser & MLC6247 (wt\%) & $\lambda_{\text {laser }}(\mathrm{nm})$ \\
\hline 1 & 28.30 & 577 \\
2 & 27.38 & 593 \\
3 & 26.94 & 603 \\
4 & 26.51 & 618 \\
5 & 26.10 & 632 \\
\hline
\end{tabular}

fluorescence emission of PM597 and DCM and consequently a higher optical efficiency.

In order to investigate the effectiveness of OBD in the performance and tunability of CLC lasers, five CLC lasers with the nematic liquid crystal BL009 and different concentrations of the chiral agent MLC6247 including $1.490 \mathrm{wt} \%$ of OBD (0.924 wt\% of DCM and $0.566 \mathrm{wt} \%$ of PM597) were prepared (Table 3).

Figure 10 illustrates the transmittance and laser line spectra of above CLC lasers. As seen, increasing the concentration of chiral impurity and consequently decreasing the pitch result in blue-shifting long-wavelength edge $\left(\lambda_{1}\right)$ of PBG. The laser wavelengths $\left(\lambda_{\text {laser }}\right)$ were measured by a spectrometer HR2000 (from Ocean Optics) with electronic and optical resolutions $0.22 \mathrm{~nm}$ and $0.65 \mathrm{~nm}$, respectively.

The spot size of pumping laser in all CLC lasers was set at $95 \mu \mathrm{m}$ by adjusting the location of lens L1 (Fig. 1). Moreover, all samples were pumped by the laser energy of $15 \mu \mathrm{J} /$ pulse. Since the laser radiation was emitted in a resonance cavity with very small diameter and length (less than 100 and 10 microns, respectively), propagation of laser at far distances undergoes a diffraction effect (Fig. 11).
Fig. 10 Normalized transmission spectra and laser lines of five CLC lasers corresponding to Table 3. All spectra were measured by a spectrometer HR2000 (from Ocean Optics)
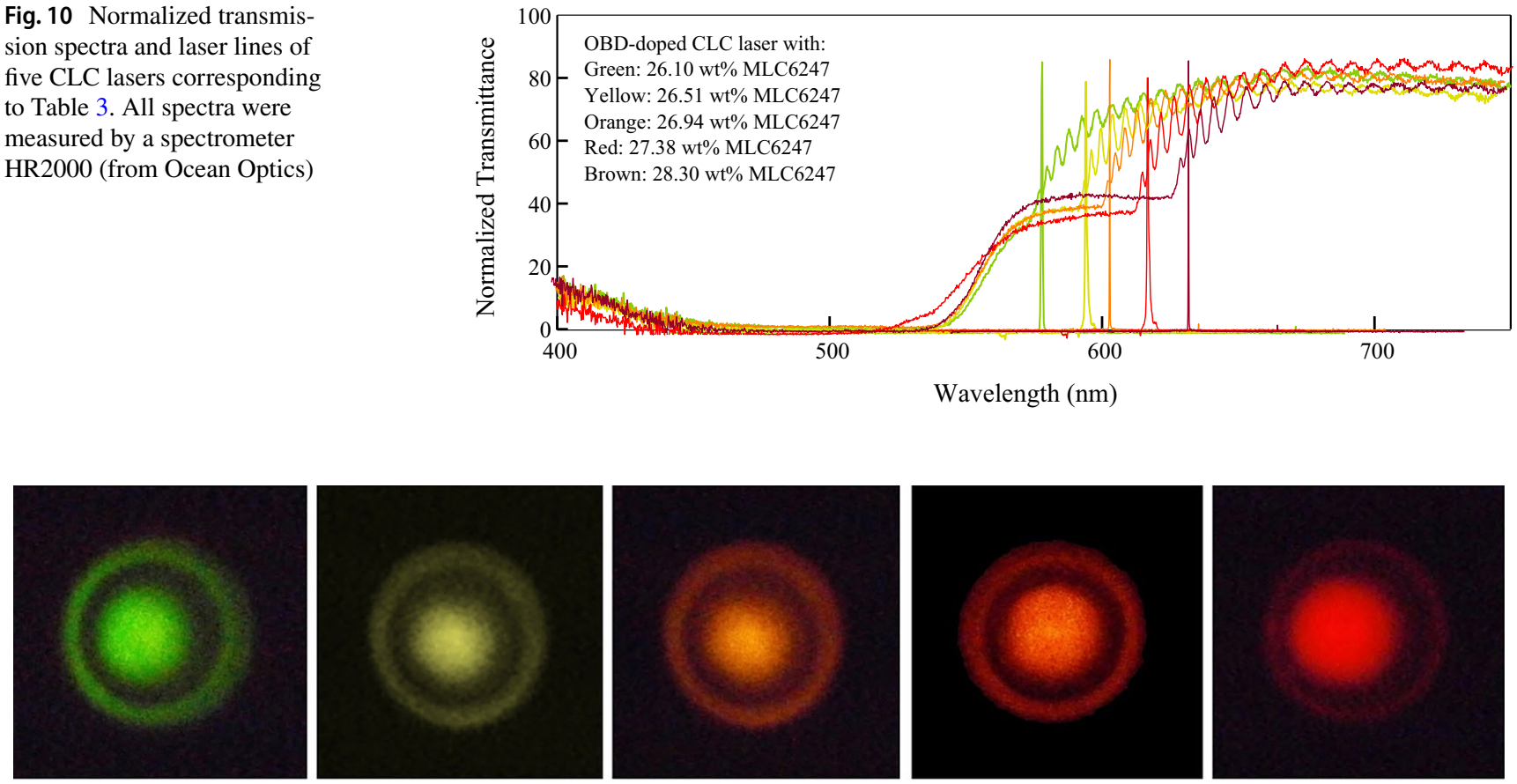

$\lambda_{1}=577 \mathrm{~nm}$

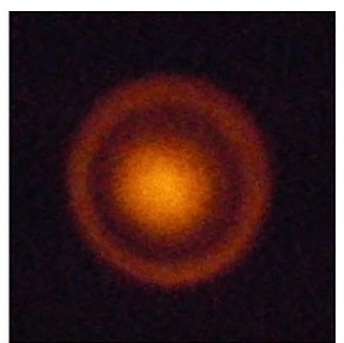

$\lambda_{3}=603 \mathrm{~nm}$

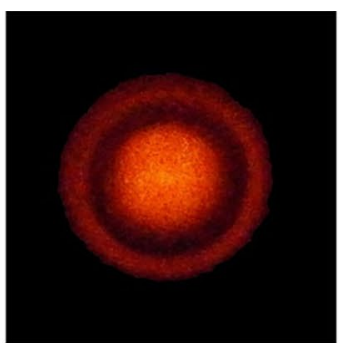

$\lambda_{4}=618 \mathrm{~nm}$

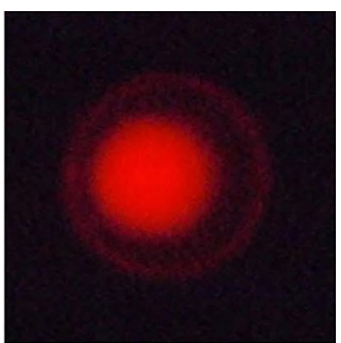

$\lambda_{5}=632 \mathrm{~nm}$

Fig. 11 Far-field diffraction pattern of five OBD-doped CLC lasers corresponding to Table 3 at $25 \mathrm{~cm}$ from the laser 


\section{Conclusion}

In this research, a method to substantially enhance the lasing efficiency of a CLC band-edge laser was investigated by utilizing an optimal binary-dye mixture comprising $62 \mathrm{wt} \%$ of DCM and 38 wt\% of PM597 as an active medium. The results indicated that the slope efficiency of an OBD-doped CLC laser is increased by $20 \%$ compared to the highest efficiency associated with DCM- and PM597-doped ones. In addition, the measurements proved that the fluorescence spectrum of OBD is wider than that of DCM and PM597. This means that OBD can be much more appropriate than each of individual dyes to fabricate a tunable CLC laser. Furthermore, the higher laser efficiency can be originated from better alignment of the transition dipole moments of DCM and PM597 with the local director of CLC as a result of effective mutual interaction between two dyes in the liquid crystal host (BL009) and also greater order parameter of PM597 in comparison with DCM.

Open Access This article is distributed under the terms of the Creative Commons Attribution 4.0 International License (http://creativeco mmons.org/licenses/by/4.0/), which permits unrestricted use, distribution, and reproduction in any medium, provided you give appropriate credit to the original author(s) and the source, provide a link to the Creative Commons license, and indicate if changes were made.

\section{References}

1. Wang, L., Urbas, A.M., Li, Q.: Nature-inspired emerging chiral liquid crystal nanostructures: from molecular self-assembly to DNA mesophase and nanocolloids. Adv. Mater. 30, 1801335 (2018)

2. Wang, L., Dong, H., Li, Y., Liu, R., Wang, Y.-F., Bisoyi, H.K., Sun, L.-D., Yan, C.-H., Li, Q.: Luminescence-driven reversible handedness inversion of self-organized helical superstructures enabled by a novel near-infrared light nanotransducer. Adv. Mater. 27, 2065 (2015)

3. Wang, L., Dong, H., Li, Y., Xue, C., Sun, L.-D., Yan, C.-H., Li, Q.: Reversible near-infrared light directed reflection in a selforganized helical superstructure loaded with upconversion nanoparticles. J. Am. Chem. Soc. 136, 4480 (2014)

4. Wang, L., Bisoyi, H.K., Zheng, Z., Gutierrez-Cuevas, K.G., Singh, G., Kumar, S., Bunning, T.J., Li, Q.: Stimuli-directed selforganized chiral superstructures for adaptive windows enabled by mesogen-functionalized graphene. Mater. Today 20, 230 (2017)

5. Wang, L., Li, Q.: Stimuli-directing self-organized 3D liquid-crystalline nanostructures: from materials design to photonic applications. Adv. Funct. Mater. 26, 10 (2016)

6. Wang, L.: Self-activating liquid crystal devices for smart laser protection. Liq. Cryst. 43, 2062 (2016)

7. Kopp, V.I., Fan, B., Vithana, H.K.M., Genack, A.Z.: Low-threshold lasing at the edge of a stop band in cholesteric liquid, crystals. Opt. Lett. 23, 1707 (1998)
8. De Gennes, P.G.: The Physics of Liquid Crystals. Clarendon Press, Oxford (1974)

9. Kopp, V.I., Genack, A.Z.: Large coherence area thin-film photonic stop-band lasers. Phys. Rev. Lett. 86, 1753 (2001)

10. Munoz, A., Muhoray, P.P., Taheri, B.: Ultraviolet lasing in cholesteric liquid crystals. Opt. Lett. 26, 804 (2001)

11. Alvarez, E., He, M., Munoz, A.F., Pallfy-Muhoray, P., Serak, S.V., Taheri, B., Twieg, R.: Mirrorless lasing and energy transfer in cholesteric liquid crystals doped with laser dyes. Mol. Cryst. Liq. Cryst. 369, 75 (2001)

12. Il'chishin, I.P., Tikhonov, E.A., Tishchenko, V.G., Shpak, M.T.: Generation of tunable radiation by impurity cholesteric liquid crystals. JETP Lett. 32, 24 (1980)

13. Zhou, Y., Huang, Y., Rapaport, A., Bass, M., Wu, S.T.: Doubling the optical efficiency of a chiral liquid crystal laser using a reflector. Appl. Phys. Lett. 87, 231107 (2005)

14. Schmidtke, J., Stille, W., Finkelmann, H., Kim, S.T.: Laser emission in a dye doped cholesteric polymer network. Adv. Mater. 14, 746 (2002)

15. Shirvani-Mahdavi, H., Mohajerani, E., Wu, S.T.: Circularly polarized high-efficiency cholesteric liquid crystal lasers with a tunable nematic phase retarder. Opt. Express 18, 5021 (2010)

16. Shirvani-Mahdavi, H., Fardad, S., Mohajerani, E., Wu, S.T.: High efficiency cholesteric liquid crystal lasers with an external stable resonator. Opt. Express 18, 13593 (2010)

17. Woon, K.L., O’Neill, M., Richards, G.J., Aldred, M.P., Kelly, S.M.: Stokes parameter studies of spontaneous emission from chiral nematic liquid crystals as a one-dimensional photonic stop-band crystal experiment and theory. Phys. Rev. E 71, 041706 (2005)

18. Morris, S.M., Ford, A.D., Pivnenko, M.N., Coles, H.J.: Enhanced emission from liquid-crystal lasers. J. Appl. Phys. 97, 023103 (2005)

19. Cao, W., Muhoray, P.P., Taheri, B., Marino, A., Abbate, G.: Lasing thresholds of cholesteric liquid crystals lasers. Mol. Cryst. Liq. Cryst. 429, 101 (2005)

20. Morris, S.M., Ford, A.D., Gillespie, C., Pivnenko, M.N., Hadeler, O., Coles, H.J.: The emission characteristics of liquid-crystal lasers. J. SID 14, 565 (2006)

21. Araoka, F., Shin, K.C., Takanishi, Y., Ishikawa, K., Zhu, Z., Swager, T.M., Takezoe, H.: How doping a cholesteric liquid crystal with polymeric dye improves an order parameter and makes possible low threshold lasing. J. Appl. Phys. 94, 279 (2003)

22. Shin, K.C., Araoka, F., Park, B., Takanishi, Y., Ishikawa, K., Zhu, Z., Swager, T.M., Takezoe, H.: Advantages of highly ordered polymer dyes for lasing in chiral nematic liquid crystals. J. Appl. Phys. 43, 631 (2004)

23. Dolgaleva, K., Wei, S.K.H., Lukishova, S.G., Chen, S.H., Schwertz, K., Boyd, R.W.: Enhanced laser performance of cholesteric liquid crystal doped with oligofluorene dye. J. Opt. Soc. Am. B 25, 1496 (2008)

24. Amemiya, K., Shin, K.C., Takanishi, Y., Ishikawa, K., Azumi, R., Takezoe, H.: Lasing in cholesteric liquid crystal doped with oligothiophene derivatives. Jpn. J. Appl. Phys. 43, 6084 (2004)

25. Huang, Y., Zhou, Y., Hong, Q., Rapaport, A., Bass, M., Wu, S.T.: Incident angle and polarization effects on the dye-doped cholesteric liquid crystal laser. Opt. Commun. 261, 91 (2006)

26. Meyer, M., Mialocq, Y.C., Perly, B.: Photoinduced intramolecular charge transfer and trans-cis isomerization of the DCM styrene dye: picosecond and nanosecond laser spectroscopy, high performance liquid chromatography and nuclear magnetic resonance studies. J. Phys. Chem. 94, 98 (1990) 
27. Schmidtke, J., Stille, W.: Fluorescence of a dye-doped cholesteric liquid crystal film in the region of the stop-band: theory and experiment. Eur. Phys. J. 31, 179 (2003)

28. Sonoyama, K., Takanishi, Y., Ishikawa, K., Takezoe, H.: Lowering threshold by energy transfer between two dyes in cholesteric liquid crystal distributed feedback lasers. Appl. Phys. Express 1, 032002 (2008)

Publisher's Note Springer Nature remains neutral with regard to jurisdictional claims in published maps and institutional affiliations. 